\title{
Renal Clearance Normalized by SA
}

National Cancer Institute

\section{Source}

National Cancer Institute. Renal Clearance Normalized by SA. NCI Thesaurus. Code C105460.

The rate at which a substance is removed from the blood via the kidneys, divided by the surface area. 